\title{
Clinical and Economic Burden of Chronic Heart Failure and Reduced Ejection Fraction Following a Worsening Heart Failure Event
}

\author{
Javed Butler • Laurence M. Djatche - Baanie Sawhney • \\ Sreya Chakladar · Lingfeng Yang · Joanne E. Brady · Mei Yang
}

Received: June 19, 2020 / Published online: August 6, 2020

(C) The Author(s) 2020

\begin{abstract}
Introduction: A worsening heart failure event (WHFE) is defined as progressively escalating heart failure signs/symptoms requiring intravenous diuretic treatment or hospitalization. No studies have compared the burden of chronic heart failure with reduced ejection fraction (HFrEF) following a WHFE versus stable disease to inform healthcare decision makers.

Methods: A retrospective study using the $\mathrm{IBM}^{\circledR}$ MarketScan ${ }^{\circledR}$ Commercial Database included patients younger than 65 years of age with HFrEF (one inpatient or two outpatient claims of systolic HF or one outpatient claim of systolic
\end{abstract}

Digital Features To view digital features for this article go to https://doi.org/10.6084/m9.figshare.12689600.

Electronic supplementary material The online version of this article (https://doi.org/10.1007/s12325020-01456-1) contains supplementary material, which is available to authorized users.

J. Butler $(\square)$

University of Mississippi Medical Center, Jackson, MS, USA

e-mail: jbutler4@umc.edu

L. M. Djatche $\cdot$ L. Yang · J. E. Brady · M. Yang

Merck \& Co., Inc., Kenilworth, NJ, USA

B. Sawhney $\cdot$ S. Chakladar

Complete Health Economics and Outcomes

Research Solutions, North Wales, PA, USA
HF plus one outpatient claim of any HF). The first claim for HFrEF during 2016 was the index date. Patients were followed for the first 12 months after the index date (the worsening assessment period) to identify a WHFE, and for an additional 12 months or until the end of continuous enrollment (the post-worsening assessment period). Mean per patient per month (PPPM) health care resource use (HCRU) and costs were compared between patients following a WHFE and stable patients during the two periods using generalized linear models adjusting for patient characteristics.

Results: Of 16,646 patients with chronic HFrEF, $26.8 \%$ developed a WHFE. Adjusted all-cause hospitalizations $(0.16$ vs. 0.02 PPPM, $P<0.0001)$, outpatient visits $(3.54$ vs. 2.73 PPPM, $P<0.0001)$, and emergency department visits $(0.25$ vs. 0.06 PPPM, $P<0.0001)$ were higher in patients following a WHFE than stable patients during the worsening assessment period. Similar differences in HCRU were observed between the two cohorts during the post-worsening assessment period. Mean total adjusted cost of care PPPM was $\$ 8657$ in patients with HFrEF following a WHFE versus \$2195 in stable patients during the worsening assessment period, and $\$ 6809$ versus $\$ 2849$, respectively, during the post-worsening assessment period.

Conclusion: HCRU and costs were significantly greater in patients with chronic HFrEF following a WHFE compared to those who remained 
stable, suggesting an unmet need to improve clinical and economic outcomes among these patients.

Keywords: Cost and cost analysis/economics; Health care resource utilization; Worsening heart failure

\section{Key Summary Points}

Why carry out this study?

Heart failure is a progressive condition and repeated hospitalizations have been associated with increased risk of mortality.

Patients who develop a worsening heart failure event (escalating heart failure signs/symptoms requiring intravenous diuretic treatment in the outpatient setting or hospitalization) have high rates of recurrent heart failure hospitalization and mortality.

In this study, we assessed the economic impact of a worsening heart failure event among a commercially insured population by performing a retrospective claims-based analysis that compared health care resource utilization and health care costs between patients with chronic HFrEF following a worsening heart failure event and patients with stable chronic HFrEF.

\section{What was learned from the study?}

This is the first study to investigate health care resource utilization and health care costs among patients aged less than 65 years with chronic HFrEF following a worsening heart failure event versus those with chronic HFrEF who remain stable.

There is a high clinical and economic burden among patients with chronic HFrEF following a worsening heart failure event compared to patients with stable chronic HFrEF, highlighting an ongoing unmet need to improve overall outcomes in patients with chronic HFrEF following a worsening heart failure event.

\section{INTRODUCTION}

The prevalence of heart failure (HF) in the USA is predicted to increase by $46 \%$ from 2012 to 2030, resulting in more than eight million adults 18 years or older having HF with nearly $\$ 70$ billion in associated health care costs [1]. HF leads to approximately one million hospitalizations annually, with more than half of these patients being readmitted within 6 months of discharge and almost 30\% dying within 1 year [2-4]. Patients with HF are typically categorized on the basis of left ventricular ejection fraction (LVEF), and the prevalence of HF with reduced ejection fraction (HFrEF) varies in the literature and is reported to range from $31 \%$ to $56 \%$ [5-11].

HFrEF is a progressive condition with substantial variation in disease trajectory among patients. While some patients remain symptomatically stable, others will develop a worsening HF event that is associated with symptom escalation that can advance to the point of requiring $\mathrm{HF}$ hospitalization or intravenous diuretic treatment in a clinical setting [12-16]. An observational cohort analysis of the PINNACLE Registry found that one in six newly diagnosed patients with HFrEF will have a worsening HF event within 18 months of HF diagnosis, and those patients have poor outcomes through 2 years [14]. In this study, 56\% of those who developed a worsening HF event were re-hospitalized within 30 days of the worsening HF event; and the 2-year mortality rate was 23\% [14]. Analysis of patient data from the Get With The Guidelines ${ }^{\circledR}$-Heart Failure registry and claims data from the US Centers for Medicare and Medicaid Services also showed higher rates of readmission for HF among patients with HFrEF. During the 5-year followup, patients with HFrEF had an HF-related readmission rate of $48.5 \%$ [6]. Mortality rates are 3-fold higher for patients hospitalized for HF compared with those who are never hospitalized, and the risk increases with each subsequent hospitalization [17, 18].

In 2015, the US Food and Drug Administration approved two new drugs for HFrEF including sacubitril/valsartan and ivabradine 
$[19,20]$. These treatments may be administered in conjunction with or in place of other HF therapies [19-21]. Given the evolving standard of care with these newer agents in 2015, it is important to evaluate the economic burden and unmet clinical need in patients with chronic HFrEF who develop a worsening HF event and survive versus those who remain stable [19-23].

Several studies have shown that there is high economic burden and health care resource utilization (HCRU) in patients with HFrEF, and a high disease burden in patients following a worsening HF event [14, 24-26]. However, no study has investigated outcomes in patients with chronic HFrEF following a worsening HF event (WHFE) versus patients with chronic HFrEF who remain stable. In this study, we assessed the economic impact of a WHFE among a commercially insured population by performing a retrospective claims-based analysis that compared HCRU and health care costs between patients with chronic HFrEF following a WHFE and patients with stable chronic HFrEF.

\section{METHODS}

\section{Study Design and Data Sources}

This retrospective claims analysis utilized data from the IBM $^{\circledR}$ MarketScan ${ }^{\circledR}$ Commercial and Medicare Supplemental Databases from January 1, 2015 to December 31, 2018 to examine patient characteristics and outcomes. The study design is shown in Fig. 1. These databases represent the health services of 174 million employees, dependents, and retirees in the USA with primary or Medicare supplemental coverage through privately insured fee-for-service, point-of-service, or capitated health plans. IBM Watson Health and MarketScan are trademarks of IBM Corporation in the USA, other countries, or both. This article reports on a retrospective, observational study using de-identified anonymized patient-level data. Therefore, informed consent and ethics committee approval were not required. Merck accessed the IBM $^{\circledR}$ MarketScan ${ }^{\circledR}$ Research Databases through a standard license agreement with IBM. Personnel from Complete Health Economics and
Outcomes Research Solutions were granted access to the data via a third-party agreement in accordance with the terms and conditions of the agreement between Merck and IBM.

\section{Study Population and Eligibility Criteria}

The population included adult patients aged at least 18 years and less than 65 years with HFrEF (prevalent or incident) for which data on HCRU and costs were fully available and covered by the commercial health insurance. The first claim of HFrEF recorded from January 1, 2016 to December 31, 2016 was defined as the HFrEF index date (Fig. 1). Patients were considered to have HFrEF if they had one hospitalization claim or two outpatient claims of systolic HF (International Statistical Classification of Diseases and Related Health Problems, 10th revision [ICD-10]: I50.2x, I50.4x, I50.1), or one outpatient claim of systolic HF plus one outpatient claim of any HF (ICD-10 I50.2x, I50.4x, I50.8x, I50.1, I50.3x, I11.0, I50.9) on two different dates within a 12-month period. Patients with evidence of adult congenital heart disease (e.g., single ventricular disease), amyloidosis, a heart transplant, or implantation of a left ventricular assist device during the 12 months prior to the HFrEF index date were excluded from the analysis.

The 12-month period before the HFrEF index date was defined as the baseline period. After the HFrEF index date, patients were followed for a period of 12 months to identify worsening HF events based on the presence of HF-related hospitalization and/or intravenous diuretic use. This 12-month period was defined as the worsening assessment period, and during this period, patients were required to have 12 months continuous enrollment after the index date in the health plan. Patients who developed a worsening HF event during this period were identified as patients with chronic HFrEF following a WHFE, and patients who did not develop a worsening HF event during this period were defined as patients with stable chronic HFrEF. Patients were followed for an additional 12 months, defined as the post-worsening assessment period. The post-worsening 


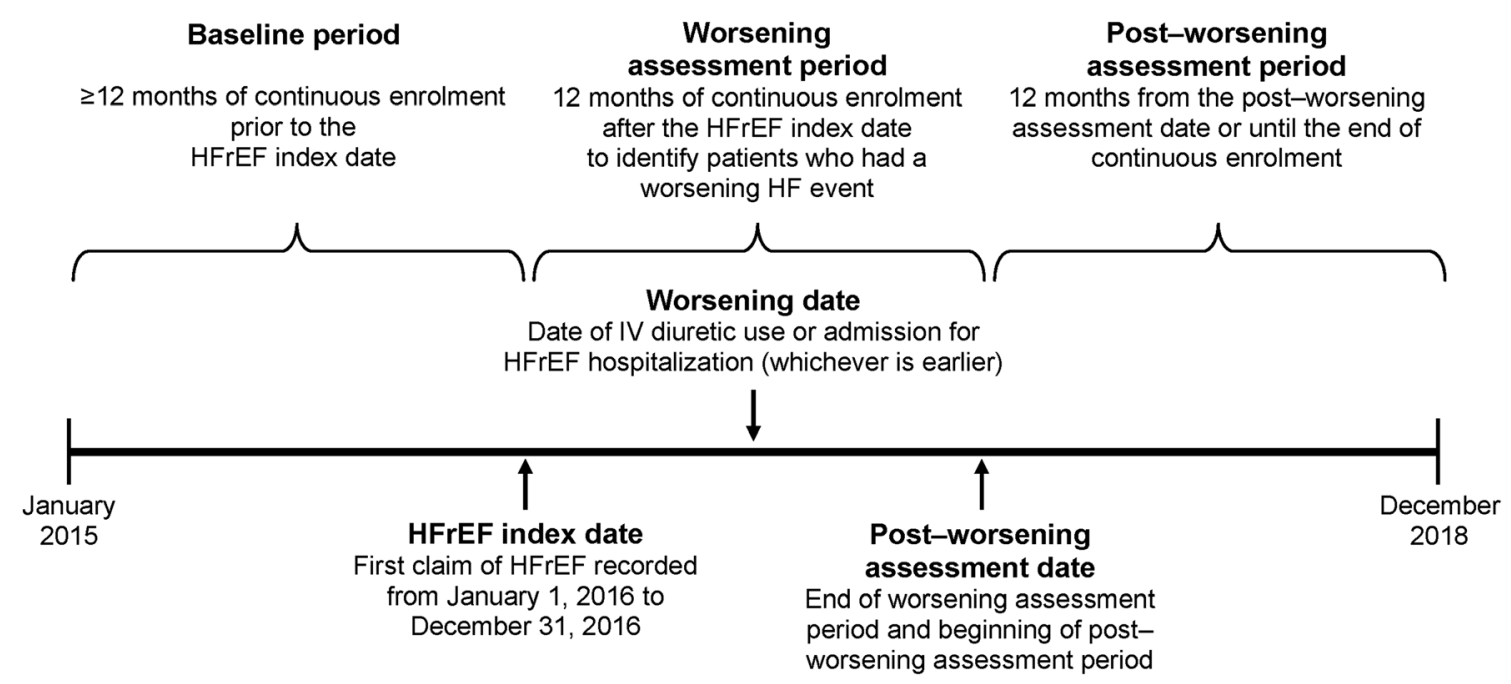

Fig. 1 Study design. The study population included adult patients aged less than 65 years who were diagnosed with prevalent or incident HFrEF. The first claim of HFrEF recorded from January 1, 2016 to December 31, 2016 was defined as the HFrEF index date. The 12-month period prior to the HFrEF index date was defined as the baseline period. The 12-month period after the HFrEF index date was defined as the worsening assessment period and was

assessment follow-up began at the end of the worsening assessment period and lasted for 12 months or until the end of continuous enrollment, whichever occurred first.

\section{Patient Characteristics}

Baseline demographics, clinical characteristics, HCRU, and associated costs were assessed during the 12-month baseline period for patients with chronic HFrEF following a WHFE and patients with stable chronic HFrEF. The exception to this method was baseline use of HF-related medications, which was assessed for the period within 90 days prior to the index date. The measures used to assess HCRU and costs are described below.

\section{Outcomes}

HCRU and costs were assessed during the worsening and post-worsening assessment periods for patients with chronic HFrEF used to identify a worsening heart failure event based on the presence of heart failure-related hospitalization and/or intravenous diuretic use. The worsening assessment period was followed by an additional 12-month follow-up defined as the post-worsening assessment period. Abbreviations: HFrEF, heart failure with reduced ejection fraction; IV, intravenous

following a WHFE and patients with stable chronic HFrEF. HCRU was assessed separately for HF-related and all-cause utilization, and by setting of care (hospitalization, outpatient, and emergency department [ED]). HCRU was measured as the mean number of visits per patient per month (PPPM). For hospitalization, utilization was also measured as the mean length of hospital stay PPPM. Similar to HCRU, health care costs were assessed for HF-related and all-cause costs. Costs were calculated in total and by setting of care (hospitalization, outpatient, ED, pharmacy, and other) as mean costs PPPM. The study was designed to capture costs from the perspective of a private payer, and reported costs represented those paid by the commercial health plan. Costs were adjusted to 2018 US dollars [27].

\section{Statistical Analysis}

Descriptive statistics were used to summarize baseline demographics, comorbidities, and 
medications. Categorical variables were summarized as the number and percentage of the total study population and by subgroups where appropriate; continuous variables were reported as mean \pm standard deviation (SD). A modified Charlson Comorbidity Index (CCI), which excluded congestive HF, was calculated using a total score and a scoring algorithm to weight each observed comorbidity $[28,29]$. $T$ tests were performed to assess between-group differences in baseline characteristics for continuous variables, and chi-square tests were performed for categorical variables.

Multivariable models were fit and compared HCRU and costs between patients with chronic HFrEF following a WHFE and patients with stable chronic HFrEF. HCRU was modeled using generalized linear models (GLMs) with negative binomial distribution or two-part/zero inflated models (where appropriate) for count outcomes, and costs were modeled using GLMs with log link function and gamma distribution or two-part models (where appropriate) [30]. Least square means (from the GLMs) were used to report adjusted mean costs [31]. The modified Park test was used to check the family of gamma distribution. The following baseline demographic and clinical characteristics were used to adjust for differences between patients with chronic HFrEF following a WHFE and patients with stable chronic HFrEF: gender, geographic region, health plan type, CCI, HF medication use within 90 days prior to the index date, and HCRU during the 12-month baseline period (all-cause hospitalization and outpatient visits).

\section{RESULTS}

\section{Patient Characteristics}

The study population included 16,646 patients with 4460 (26.8\%) developing a worsening HF event during the worsening assessment period (Fig. S1 in the electronic supplementary material for details). Demographic characteristics were similar between patients with chronic HFrEF following a WHFE and patients with stable chronic HFrEF. The mean age was
56.1 years for patients with chronic HFrEF following a WHFE and 55.4 years for patients with stable chronic HFrEF, and $63.7 \%$ of patients with chronic HFrEF following a WHFE versus $64.6 \%$ of patients with stable chronic HFrEF were male (Table 1). More than half of the patients in each group were covered by a preferred provider organization $(50.5 \%$ of patients with chronic HFrEF following a WHFE, $54.4 \%$ of patients with stable chronic HFrEF).

Patients with chronic HFrEF following a WHFE had more comorbidities at baseline, indicated by a higher mean modified CCI score and a higher prevalence of multiple cardiovascular comorbidities including atrial fibrillation, coronary artery disease, type 2 diabetes, hyperlipidemia, hypertension, and myocardial infarction (Table 1). HF-related medication prescriptions within 90 days prior to the index date were similar between the two groups, except in the case of angiotensin-converting enzyme inhibitors (ACEi)/angiotensin II receptor blockers (ARBs) and diuretics (Table 1). Few patients in the study population received angiotensin receptor neprilysin inhibitors $(1.3 \%$ of patients with stable chronic HFrEF, $1.2 \%$ of patients with chronic HFrEF following a WHFE) or ivabradine ( $0.3 \%$ in both groups).

HF-related HCRU PPPM during the baseline period was higher in patients with chronic HFrEF following a WHFE versus patients with stable chronic HFrEF (Table 1): mean \pm SD $0.04 \pm 0.09$ vs. $0.02 \pm 0.04$ HF-related hospitalizations, $0.24 \pm 0.48$ vs. $0.18 \pm 0.33$ outpatient visits, and $0.04 \pm 0.09$ vs. $0.01 \pm 0.04 \mathrm{ED}$ visits, respectively. Similar trends were observed for all-cause HCRU.

Costs PPPM were higher for patients with chronic HFrEF following a WHFE compared with patients with stable chronic HFrEF during the baseline period (Table 1): mean total HFrelated cost was $\$ 942 \pm \$ 3018$ vs. $\$ 454 \pm \$ 1882$, respectively; and mean total allcause cost was $\$ 4676 \pm \$ 8751$ vs. $\$ 2830 \pm \$ 5971$, respectively. 
Table 1 Baseline patient demographic, clinical, and treatment characteristics

\begin{tabular}{|c|c|c|c|}
\hline Characteristics & $\begin{array}{l}\text { Patients with stable } \\
\text { chronic HFrEF } \\
(N=12,186)\end{array}$ & $\begin{array}{l}\text { Patients with chronic } \\
\text { HFrEF following a worsening } \\
\text { HF event }(N=4460)\end{array}$ & $P$ value \\
\hline Age, mean $(\mathrm{SD})$ years & $55.4(8.2)$ & $56.1(8.0)$ & $<0.0001$ \\
\hline Gender (\%) & & & 0.2728 \\
\hline Male & 64.6 & 63.7 & \\
\hline Geographic region (\%) & & & 0.0098 \\
\hline Northeast & 17.9 & 16.8 & \\
\hline North central & 20.9 & 23.4 & \\
\hline South & 50.9 & 50.0 & \\
\hline West & 10.2 & 9.7 & \\
\hline Other \& unknown & 0.1 & 0.1 & \\
\hline Plan type (\%) & & & $<0.0001$ \\
\hline $\mathrm{PPO}$ & 54.4 & 50.5 & \\
\hline $\mathrm{CDHP} / \mathrm{HDHP}$ & 17.9 & 18.1 & \\
\hline $\mathrm{HMO}$ & 9.6 & 10.2 & \\
\hline Comprehensive & 6.7 & 9.0 & \\
\hline POS/POS with capitation & 8.8 & 8.6 & \\
\hline Other and unknown & 2.6 & 3.7 & \\
\hline Primary payer (\%) & & & $<0.0001$ \\
\hline Medicare supplemental & 2.1 & 3.5 & \\
\hline Commercial & 97.9 & 96.5 & \\
\hline \multicolumn{4}{|l|}{ Comorbidities (\%) } \\
\hline Anemia & 11.5 & 20.2 & $<0.0001$ \\
\hline Atrial fibrillation & 20.5 & 28.6 & $<0.0001$ \\
\hline Chronic kidney disease & 13.6 & 26.8 & $<0.0001$ \\
\hline Coronary artery disease & 34.2 & 41.5 & $<0.0001$ \\
\hline Type 2 diabetes & 35.1 & 49.7 & $<0.0001$ \\
\hline Hyperlipidemia & 56.2 & 61.0 & $<0.0001$ \\
\hline Hypertension & 72.5 & 80.6 & $<0.0001$ \\
\hline Myocardial infarction & 28.0 & 33.6 & $<0.0001$ \\
\hline Sleep apnea & 21.4 & 27.4 & $<0.0001$ \\
\hline History of HF & 55.5 & 63.1 & $<0.0001$ \\
\hline Modified CCI score, mean (SD) & $3.1(2.2)$ & $4.2(2.7)$ & $<0.0001$ \\
\hline
\end{tabular}


Table 1 continued

\begin{tabular}{ll}
\hline Characteristics & Patients with stable \\
& chronic HFrEF \\
& $(N=12,186)$
\end{tabular}

Patients with chronic $\quad P$ value
HFrEF following a worsening
HF event $(N=4460)$

HF-related medication within 90 days, ${ }^{a}$ (\%)

\begin{tabular}{|c|c|c|c|}
\hline ACEi/ARB & 57.5 & 50.9 & $<0.0001$ \\
\hline Beta blockers & 62.5 & 61.0 & 0.0755 \\
\hline Diuretics & 42.2 & 55.2 & $<0.0001$ \\
\hline Aldosterone antagonist & 19.8 & 18.1 & 0.0179 \\
\hline $\mathrm{ARNi}$ & 1.3 & 1.2 & 0.5004 \\
\hline Digoxin & 7.1 & 7.6 & 0.3008 \\
\hline Ivabradine & 0.3 & 0.3 & 0.6798 \\
\hline \multicolumn{4}{|c|}{ Other medication within 12 months, ${ }^{\mathrm{b}}$ (\%) } \\
\hline Hydralazine plus nitrate & 10.4 & 18.1 & $<0.0001$ \\
\hline Hydralazine monotherapy & 6.0 & 11.1 & $<0.0001$ \\
\hline Long-acting nitrates & 12.5 & 17.5 & $<0.0001$ \\
\hline Statins & 43.5 & 46.0 & 0.0045 \\
\hline GLP-1 RA & 3.0 & 3.8 & 0.0069 \\
\hline SGLT2i & 2.8 & 2.8 & 0.8828 \\
\hline DPP4i & 5.6 & 7.2 & 0.0001 \\
\hline Sulfonylurea & 8.7 & 11.5 & $<0.0001$ \\
\hline Metformin & 18.9 & 21.7 & $<0.0001$ \\
\hline
\end{tabular}

HCRU, mean (SD) PPPM

HF-related

Hospitalizations

Length of hospital stay, days

Outpatient visits

ED visits

All cause

Hospitalizations

Length of hospital stay, days

Outpatient visits

ED visits
$0.02(0.04)$

$1.2(5.2)$

$0.18(0.33)$

$0.01(0.04)$

$0.04(0.07)$

$$
2.4(8.3)
$$

1.48 (1.42)

$0.07(0.14)$
$0.04(0.09)$

3.3 (9.7)

0.24 (0.48)

0.04 (0.09)

$0.08(0.13)$

$5.6(13.5)$

$1.90(1.70)$

$0.16(0.26)$

Health care costs, mean (SD) \$ PPPM

HF-related 
Table 1 continued

\begin{tabular}{|c|c|c|c|}
\hline Characteristics & $\begin{array}{l}\text { Patients with stable } \\
\text { chronic HFrEF } \\
(N=12,186)\end{array}$ & $\begin{array}{l}\text { Patients with chronic } \\
\text { HFrEF following a worsening } \\
\text { HF event }(N=4460)\end{array}$ & $P$ value \\
\hline Total & 454 (1882) & 942 (3018) & \\
\hline Hospitalization & $269(1610)$ & $668(2656)$ & \\
\hline Outpatient & $141(795)$ & $191(996)$ & \\
\hline ED & $13(92)$ & $41(233)$ & \\
\hline Pharmacy & $18(48)$ & $20(79)$ & \\
\hline Other $^{c}$ & $17(131)$ & 37 (298) & \\
\hline \multicolumn{4}{|l|}{ All cause } \\
\hline Total & $2830(5971)$ & 4676 (8751) & \\
\hline Hospitalization & $1196(4301)$ & $2317(6511)$ & \\
\hline Outpatient & 906 (2207) & $1181(2717)$ & \\
\hline ED & $77(310)$ & $158(480)$ & \\
\hline Pharmacy & 407 (1313) & $593(1626)$ & \\
\hline Other $^{c}$ & $254(1781)$ & 442 (2019) & \\
\hline
\end{tabular}

$A C E i$ angiotensin-converting enzyme inhibitor, $A R B$ angiotensin II receptor blocker, $A R N i$ angiotensin receptor neprilysin inhibitor, CCI Charlson Comorbidity Index, CDHP consumer-driven health plan, DPP4i dipeptidyl peptidase 4 inhibitor, $E D$ emergency department, GLP-1 RA glucagon-like peptide 1 receptor agonist, $H C R U$ health care resource utilization, $H D H P$ health deductible health plan, $H F$ heart failure, $H F r E F$ heart failure with reduced ejection fraction, $H M O$ health maintenance organization, $P O S$ point of service, $P P O$ preferred provider organization, $P P P M$ per patient per month, $S D$ standard deviation, $S G L T 2 i$ sodium-glucose cotransporter 2 inhibitor

a Data are for patients who received any of the listed medications for HF within 90 days prior to the index date

b Data are for patients who received any of the listed medications during the full 1-year pre-index baseline period

c Costs in Other category were incurred from the following settings or types of care: telehealth nursing facility, skilled nursing facility, school, custodial care facility, homeless shelter, hospice, adult living care facility, Indian Health Service freestanding facility, Indian Health Service provider-based facility, tribal free-standing facility, tribal provider-based facility, ambulance (land), ambulance (air or water), independent clinic, prison-correctional facility, federally qualified health center, inpatient psychiatric facility, patient home, psychiatric facility, partial hospitalization, assisted living facility, community mental health center, group home, intermediate care facility for individuals with intellectual disability, mobile unit, residential substance abuse facility, temporary lodging, psychiatric residential treatment center, walk-in retail health clinic, nonresidential substance abuse facility, place of employment/worksite, mass immunization center, outpatient hospital off campus, comprehensive inpatient rehabilitation facility, comprehensive outpatient rehabilitation facility, end-stage renal disease facility, state/local public health clinic, rural health clinic, independent laboratory, birthing center, military treatment facility, inpatient long-term care, other inpatient care, and other/unknown

\section{Outcomes}

\section{Unadjusted HCRU During the Worsening and Post-Worsening Assessment Periods}

Unadjusted HCRU was higher for patients with chronic HFrEF following a WHFE compared with patients with stable chronic HFrEF during both the worsening and post-worsening assessment periods (Table 2). A comparison of HF-related hospitalizations was not performed during the worsening assessment period because, by definition, only patients with chronic HFrEF 
Table 2 Unadjusted HCRU and costs during the worsening and post-worsening assessment periods

\begin{tabular}{|c|c|c|c|c|c|c|}
\hline & \multicolumn{3}{|c|}{ Worsening assessment period } & \multicolumn{3}{|c|}{ Post-worsening assessment period } \\
\hline & $\begin{array}{l}\text { Patients with } \\
\text { stable chronic } \\
\text { HFrEF } \\
(N=12,186)\end{array}$ & $\begin{array}{l}\text { Patients with } \\
\text { chronic HFrEF } \\
\text { following a } \\
\text { worsening HF } \\
\text { event }(N=4460)\end{array}$ & $P$ value & $\begin{array}{l}\text { Patients with } \\
\text { stable chronic } \\
\text { HFrEF } \\
(N=12,186)\end{array}$ & $\begin{array}{l}\text { Patients with } \\
\text { chronic HFrEF } \\
\text { following a } \\
\text { worsening HF } \\
\text { event }(N=\mathbf{4 4 6 0})\end{array}$ & $P$ value \\
\hline $\begin{array}{l}\text { Duration of } \\
\text { study period, } \\
\text { mean }(\mathrm{SD}) \\
\text { days }\end{array}$ & $-{ }^{\mathrm{a}}$ & $-{ }^{a}$ & & $329(86)$ & $310(103)$ & - \\
\hline \multicolumn{7}{|c|}{ HF-related HCRU, mean (SD) PPPM } \\
\hline Hospitalizations & $-{ }^{\mathrm{b}}$ & $-{ }^{\mathrm{b}}$ & $-{ }^{\mathrm{b}}$ & $0.02(0.01)$ & $0.08(0.03)$ & $<0.0001$ \\
\hline $\begin{array}{l}\text { Length of } \\
\text { hospital stay }\end{array}$ & $-{ }^{\mathrm{b}}$ & $-{ }^{\mathrm{b}}$ & $-{ }^{\mathrm{b}}$ & $0.15(0.07)$ & $0.68(0.27)$ & $<0.0001$ \\
\hline $\begin{array}{l}\text { Outpatient } \\
\text { visits }\end{array}$ & $1.33(0.36)$ & $1.65(0.50)$ & $<0.0001$ & $1.23(0.32)$ & $1.46(0.41)$ & $<0.0001$ \\
\hline ED visits & $0.008(0.002)$ & $0.093(0.041)$ & $<0.0001$ & $0.02(0.01)$ & $0.07(0.02)$ & $<0.0001$ \\
\hline \multicolumn{7}{|c|}{ All-cause HCRU, mean (SD) PPPM } \\
\hline Hospitalizations & $0.02(0.01)$ & $0.16(0.01)$ & $<0.0001$ & $0.04(0.02)$ & $0.13(0.05)$ & $<0.0001$ \\
\hline $\begin{array}{l}\text { Length of } \\
\text { hospital stay, } \\
\text { days }\end{array}$ & $0.28(0.10)$ & $1.43(0.06)$ & $<0.0001$ & $0.27(0.13)$ & $1.00(0.42)$ & $<0.0001$ \\
\hline $\begin{array}{l}\text { Outpatient } \\
\text { visits }\end{array}$ & $2.77(1.26)$ & $3.89(1.77)$ & $<0.0001$ & $2.51(1.27)$ & $3.31(1.91)$ & $<0.0001$ \\
\hline ED visits & $0.06(0.02)$ & $0.24(0.01)$ & $<0.0001$ & $0.07(0.02)$ & $0.20(0.07)$ & $<0.0001$ \\
\hline \multicolumn{7}{|c|}{ HF-related costs, mean (SD) \$ PPPM } \\
\hline Total & $363(596)$ & $3446(5658)$ & $<0.0001$ & $595(1215)$ & $1946(3984)$ & $<0.0001$ \\
\hline Hospitalization & $-{ }^{\mathrm{b}}$ & $-{ }^{\mathrm{b}}$ & $--^{b}$ & $441(170)$ & $1585(444)$ & 0.0003 \\
\hline Outpatient & $295(542)$ & $494(909)$ & $<0.0001$ & $143(283)$ & $297(592)$ & $<0.0001$ \\
\hline ED & $9(3)$ & $86(15)$ & $<0.0001$ & $17(6)$ & $71(23)$ & $<0.0001$ \\
\hline Pharmacy & $34(4)$ & $42(3)$ & 0.0017 & $42(6)$ & $52(7)$ & $<0.0001$ \\
\hline Other $^{c}$ & $27(3)$ & $135(11)$ & $<0.0001$ & $14(4)$ & $91(26)$ & $<0.0001$ \\
\hline \multicolumn{7}{|c|}{ All-cause costs, mean (SD) \$ PPPM } \\
\hline Total & $2428(3034)$ & $10,470(13,086)$ & $<0.0001$ & $2991(4731)$ & $7944(12,582)$ & $<0.0001$ \\
\hline Hospitalization & $467(238)$ & $6434(276)$ & $<0.0001$ & $1534(715)$ & $4984(1940)$ & $<0.0001$ \\
\hline Outpatient & $1182(34)$ & $2162(46)$ & $<0.0001$ & $789(1251)$ & $1549(2456)$ & $<0.0001$ \\
\hline ED & $62(17)$ & $244(23)$ & $<0.0001$ & $75(25)$ & $213(70)$ & $<0.0001$ \\
\hline
\end{tabular}


Table 2 continued

\begin{tabular}{|c|c|c|c|c|c|c|}
\hline & \multicolumn{3}{|c|}{ Worsening assessment period } & \multicolumn{3}{|c|}{ Post-worsening assessment period } \\
\hline & $\begin{array}{l}\text { Patients with } \\
\text { stable chronic } \\
\text { HFrEF } \\
(N=12,186)\end{array}$ & $\begin{array}{l}\text { Patients with } \\
\text { chronic HFrEF } \\
\text { following a } \\
\text { worsening HF } \\
\text { event }(N=4460)\end{array}$ & $P$ value & $\begin{array}{l}\text { Patients with } \\
\text { stable chronic } \\
\text { HFrEF } \\
(N=12,186)\end{array}$ & $\begin{array}{l}\text { Patients with } \\
\text { chronic HFrEF } \\
\text { following a } \\
\text { worsening HF } \\
\text { event }(N=4460)\end{array}$ & $P$ value \\
\hline Pharmacy & $507(23)$ & $735(27)$ & $<0.0001$ & $525(23)$ & $729(27)$ & $<0.000$ \\
\hline Other ${ }^{c}$ & 327 (27) & $943(65)$ & $<0.0001$ & $281(48)$ & $1000(216)$ & $<0.000$ \\
\hline
\end{tabular}

$E D$ emergency department, $H C R U$ health care resource utilization, $H F$ heart failure, $H F r E F$ heart failure with reduced ejection fraction, $P P P M$ per patient per month, $S D$ standard deviation

${ }^{a}$ By definition, the worsening assessment period had a duration of 12 months ( 365 days)

b By definition, only patients with chronic HFrEF following a worsening HF event could have had an HF-related hospitalization during the worsening assessment period; therefore, a comparative assessment of number of hospitalizations during this period was not performed between patients with stable chronic HFrEF and patients with chronic HFrEF following a worsening HF event

${ }^{c}$ Costs in Other category were incurred from the following settings or types of care: telehealth nursing facility, skilled nursing facility, school, custodial care facility, homeless shelter, hospice, adult living care facility, Indian Health Service freestanding facility, Indian Health Service provider-based facility, tribal free-standing facility, tribal provider-based facility, ambulance (land), ambulance (air or water), independent clinic, prison-correctional facility, federally qualified health center, inpatient psychiatric facility, patient home, psychiatric facility, partial hospitalization, assisted living facility, community mental health center, group home, intermediate care facility for individuals with intellectual disability, mobile unit, residential substance abuse facility, temporary lodging, psychiatric residential treatment center, walk-in retail health clinic, nonresidential substance abuse facility, place of employment/worksite, mass immunization center, outpatient hospital off campus, comprehensive inpatient rehabilitation facility, comprehensive outpatient rehabilitation facility, end-stage renal disease facility, state/local public health clinic, rural health clinic, independent laboratory, birthing center, military treatment facility, inpatient long-term care, other inpatient care, and other/unknown

following a WHFE could have had an HF-related hospitalization. During the worsening assessment period, HF-related HCRU was significantly different for outpatient visits and ED visits $(P<0.0001$; Table 2). All-cause HCRU was significantly different for all settings of care $(P<0.0001$; Table 2$)$. These trends were similar during the post-worsening assessment period, which had a mean (SD) duration of 310 (103) days for patients with chronic HFrEF following a WHFE and 329 (86) days for patients with stable chronic HFrEF. During this period, both HF-related and all-cause HCRU in all settings of care were significantly higher in patients with chronic HFrEF following a WHFE than in patients with stable chronic HFrEF $(P<0.0001$; Table 2). As an example, mean HF-related hospitalizations were four times as frequent in patients with a WHFE vs. stable patients $(0.08$ vs. 0.02 ; Table 2 ).

\section{Unadjusted Costs During the Worsening and Post-Worsening Assessment Periods}

Unadjusted costs were significantly higher for patients with chronic HFrEF following a WHFE compared with patients with stable chronic HFrEF during both the worsening and postworsening assessment periods $(P<0.0001$; Table 2). The main driver of total HF-related cost PPPM in the post-worsening assessment period was the HF-related hospitalization cost PPPM, which was $\$ 1585$ for patients with chronic HFrEF following a WHFE and $\$ 441$ for patients with stable chronic HFrEF. Therefore, the cost of an HF hospitalization ranged between $\$ 19,813 \quad(\$ 1585 \quad$ PPPM/0.08 


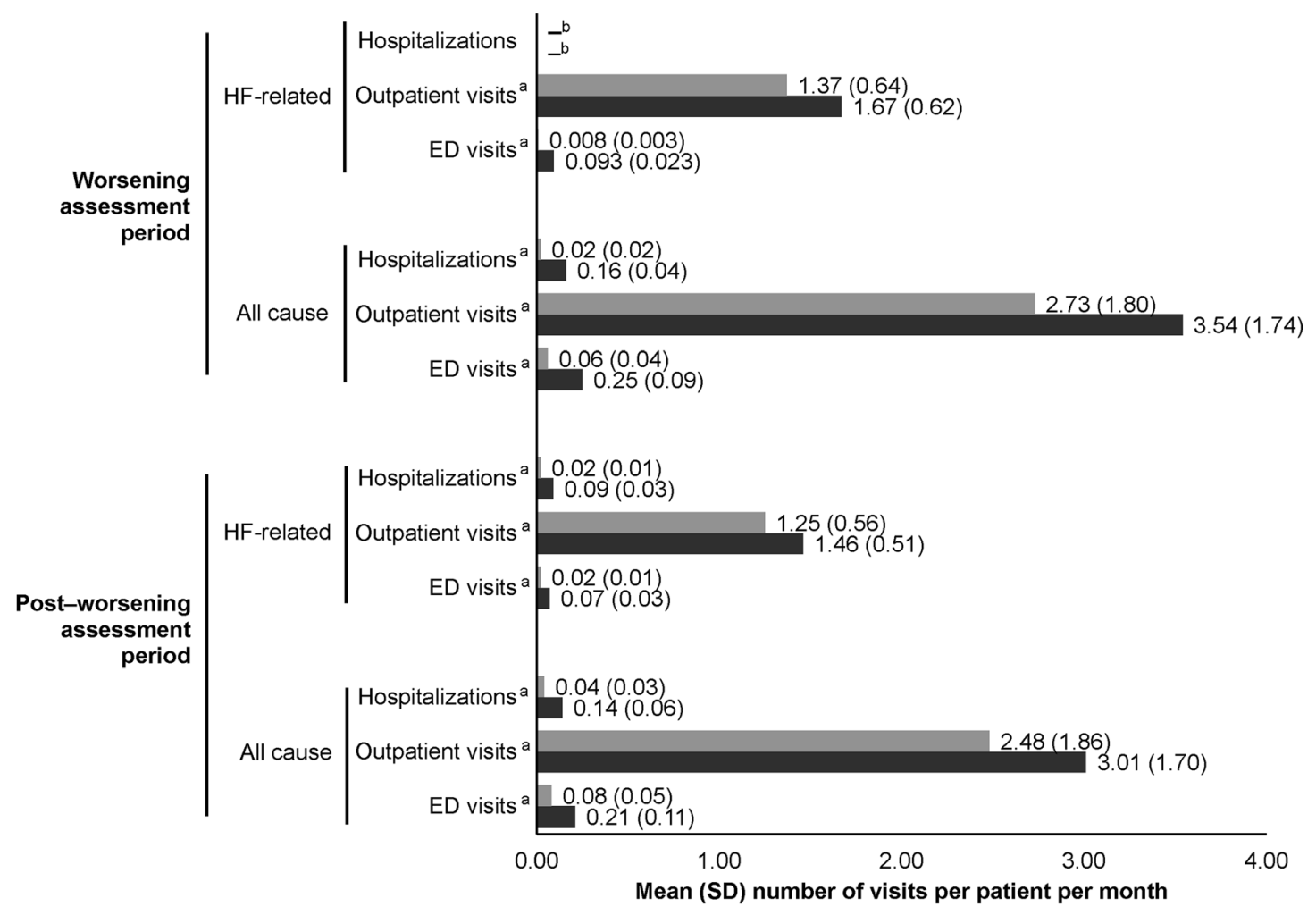

Fig. 2 Adjusted HCRU in the worsening and postworsening assessment periods. HCRU was reported by setting of care (hospitalization, outpatient, and emergency department) for patients with stable chronic HFrEF versus patients with chronic HFrEF following a worsening HF event, and reported separately for HF-related and all causerelated services. HCRU data were adjusted with the following baseline demographic and clinical characteristics: gender, geographic region, health plan type, modified Charlson Comorbidity Index, HF medication use within 90 days prior to the index date, and HCRU during the 12-month baseline period (all-cause hospitalizations and outpatient visits). Abbreviations: ED, emergency

hospitalizations PPPM) and \$22,050 (\$441 PPPM/0.02 hospitalizations PPPM) using the mean number of HF-related hospitalizations PPPM.

\section{Adjusted HCRU During the Worsening and Post-Worsening Assessment Periods} After adjustment for multiple baseline characteristics, HCRU remained higher in patients department; HF, heart failure; HFrEF, heart failure with reduced ejection fraction; HCRU, health care resource utilization; SD, standard deviation. ${ }^{a} P<0.0001$ for difference between patients with stable chronic HFrEF and patients with chronic HFrEF following a worsening HF event. ${ }^{b}$ By definition, only patients with chronic HFrEF following a worsening HF event could have had an HF-related hospitalization during the worsening assessment period; therefore, a comparative assessment of number of hospitalizations during this period was not performed between patients with stable chronic HFrEF and patients with chronic HFrEF following a worsening HF event

with chronic HFrEF following a WHFE than in patients with stable chronic HFrEF (Fig. 2). Adjusted mean HCRU was higher in the former group in both assessment periods, for both HFrelated and all-cause HCRU, and across all care settings ( $P<0.0001$ for all; Fig. 2$)$. For example, adjusted HF-related ED visits were 11.6 times more frequent in patients with a WHFE in the worsening assessment period (0.093 vs. 0.008) 


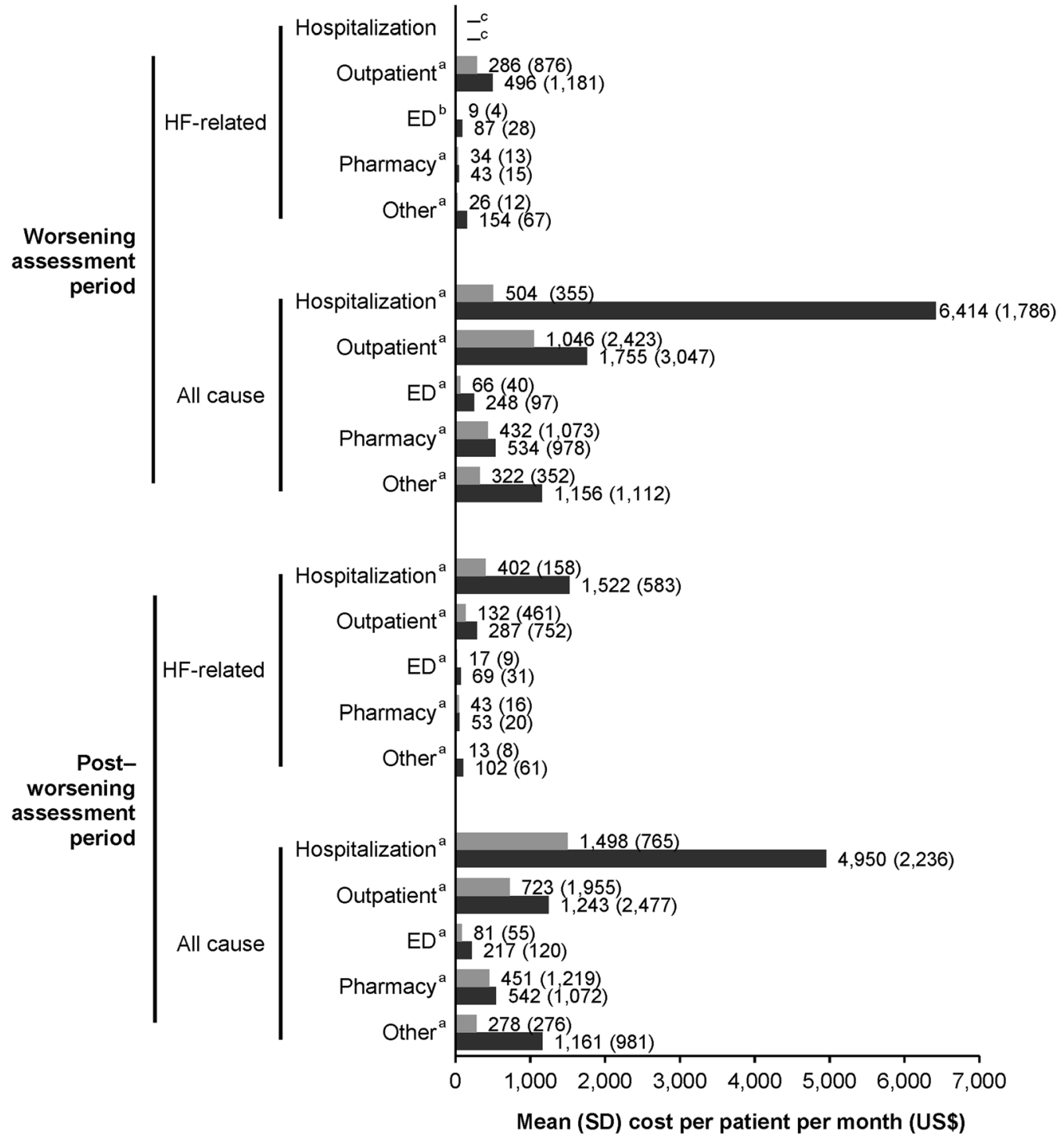

Fig. 3 Adjusted costs in the worsening and post-worsening assessment periods. Costs are reported by setting of care (hospitalization, outpatient, emergency department, pharmacy, and other) for patients with stable chronic HFrEF versus patients with chronic HFrEF following a worsening HF event, and reported separately for HF-related and all cause-related services. Costs were adjusted with the following baseline demographic and clinical characteristics: gender, geographic region, health plan type, modified Charlson Comorbidity Index, HF medication use within 90 days prior to the index date, and HCRU during the 12-month baseline period (all-cause inpatient and outpatient visits). Abbreviations: ED, emergency department; HCRU, health care resource utilization; HF, heart failure; HFrEF, heart failure with reduced ejection fraction; SD, standard deviation; US\$, United States dollars. ${ }^{\mathrm{a} P}<0.0001$ for difference between patients with stable chronic HFrEF and patients with chronic HFrEF following a worsening HF event. ${ }^{\mathrm{b}} P=0.0006$ for difference between patients with stable chronic HFrEF and patients with chronic HFrEF following a worsening $\mathrm{HF}$ event. ${ }^{\mathrm{c}} \mathrm{By}$ definition, only patients with chronic HFrEF following a worsening HF event could have had an HF-related hospitalization during the worsening assessment period; therefore, a comparative assessment of number of hospitalizations during this period was not performed between patients with stable chronic HFrEF and patients with chronic HFrEF following a worsening HF event 
and 3.5 times more frequent in the post-worsening assessment period (0.07 vs. 0.02). Adjusted mean HF-related hospitalizations during the post-worsening assessment period remained similar to their unadjusted values (0.09 in worsening patients vs. 0.02 in stable patients). For all-cause HCRU in the worsening assessment period, patients with chronic HFrEF following a WHFE were hospitalized more often than patients with stable chronic HFrEF (adjusted mean PPPM of $0.16 \pm 0.04$ hospitalizations, compared with $0.02 \pm 0.02(P<0.0001)$ (Fig. 2). Likewise, patients with stable chronic HFrEF had fewer all-cause ED visits compared with patients with chronic HFrEF following a WHFE (adjusted mean PPPM $0.06 \pm 0.04$ vs. $0.25 \pm 0.09$ visits, $P<0.0001)$. Trends were similar in the post-worsening assessment period: all-cause HCRU was greater for patients with chronic HFrEF following a WHFE versus patients with stable chronic HFrEF, with more hospitalizations (adjusted mean PPPM $0.14 \pm 0.06$ vs. $0.04 \pm 0.03, P<0.0001)$ and more ED visits (adjusted mean PPPM $0.21 \pm 0.11 \quad$ vs. $\quad 0.08 \pm 0.05, \quad P<0.0001)$ (Fig. 2).

\section{Adjusted Costs During the Worsening and Post-Worsening Assessment Periods}

The adjusted mean total HF-related cost of care PPPM during the worsening assessment period was $\$ 383 \pm \$ 1064$ for patients with stable chronic HFrEF and $\$ 3647 \pm \$ 7636$ for patients with chronic HFrEF following a WHFE $(P<0.0001)$. The mean total all-cause cost of care PPPM was $\$ 2195 \pm \$ 4572$ for patients with stable chronic HFrEF and $\$ 8657 \pm \$ 13,150$ for patients with chronic HFrEF following a WHFE $(P<0.0001)$. The primary driver of all-cause costs was hospitalization for patients with chronic HFrEF following a WHFE, while the primary driver of all-cause costs was outpatient visits in patients with stable chronic HFrEF (Fig. 3).

The mean total HF-related cost PPPM during the post-worsening assessment period was $\$ 628 \pm \$ 2301$ for patients with stable chronic HFrEF and $\$ 2044 \pm \$ 5610$ for patients with chronic HFrEF following a WHFE $(P<0.0001)$. The mean total all-cause cost PPPM was
\$2849 $\pm \$ 7681$ for patients with stable chronic HFrEF and $\$ 6809 \pm \$ 13,728$ for patients with chronic HFrEF following a WHFE $(P<0.0001)$. The primary driver of both HF-related and allcause costs was hospitalization among both patients with stable chronic HFrEF and patients with chronic HFrEF following a WHFE (Fig. 3). Comparisons of costs between patients with chronic HFrEF following a WHFE and patients with stable chronic HFrEF did not vary with adjustment in the worsening or post-worsening assessment periods.

\section{DISCUSSION}

The present report represents the first study to retrospectively assess claims-based data on commercially insured adult patients younger than age 65 years with chronic HFrEF and to compare HCRU and costs between patients following a WHFE versus those with chronic HFrEF who remained stable. The study design supported an evaluation of HCRU and cost trends for a period during which patients with chronic HFrEF could have developed a WHFE (worsening assessment period) and then a subsequent 12-month follow-up period (post-worsening assessment period). The study was designed from the perspective of a private payer and the population was restricted to commercially insured patients aged younger than 65 years.

This study showed that for commercially insured patients, HCRU and costs were higher in patients with chronic HFrEF following a WHFE versus patients with stable chronic HFrEF during both the worsening assessment and post-worsening assessment periods. This was true in the unadjusted analysis and also after adjusting for multiple variables, including gender, geographic region, health plan type, modified CCI, HF medication use within 90 days prior to the index date, and HCRU during the 12 -month baseline period. The same results were observed after adjustment of patient characteristics, suggesting that a WHFE is associated with increased clinical and economic burden as indicated by increased HCRU and associated costs, during both the period of 
worsening and for an extended period after the WHFE.

At baseline, patients with chronic HFrEF following a WHFE had higher comorbidity burden compared with patients with stable chronic HFrEF, as indicated by a higher mean modified CCI score and a higher prevalence of comorbidities associated with progression of HF including chronic kidney disease, type 2 diabetes, coronary artery disease, and hypertension. Even with more comorbidities at baseline among the higher risk group of patients with chronic HFrEF following a WHFE, use of HF-related medications within 90 days prior to the index date was generally similar except for ACEi/ARBs and diuretics. The use of ACEi/ARBs at baseline was lower among patients with chronic HFrEF following a WHFE versus patients with stable chronic HFrEF, for whom diuretic use was higher. The low use of ACEi/ARB, a recommended guideline therapy for chronic HFrEF by the American College of Cardiology Foundation/American Heart Association/Heart Failure Society of America [21], may have led to the higher likelihood of a worsening event and likely represents either clinician concern of an adverse effect (e.g., worsening renal function) or tolerability and safety (e.g., hypotension) among this highly comorbid patient population. In addition, diuretics are often used to manage HF symptoms such as edema. The higher use of diuretics at baseline in patients with chronic HFrEF following a WHFE may suggest that patients with chronic HFrEF experience symptoms and disease progression at baseline leading to HF hospitalization and a need for IV diuretics. Moreover, less renin-angiotensin-aldosterone system inhibitor therapy and the need for more diuretic use to offset HF symptoms, despite the risks associated with diuretics, highlight the challenges associated with managing patients with chronic HFrEF following a WHFE and the need for novel therapeutic options with better efficacy and safety profiles.

This study required that patients had 12 months of continuous enrollment after the HFrEF index date, which meant that all included patients had survived through at least the 12-month worsening assessment period. This design contrasts with other studies that included patients who died during the period immediately following diagnosis because of the availability of mortality data, and who therefore would have incurred higher costs [32, 33]. A retrospective claims study from a large US health plan (commercial and Medicare Advantage with Part D) showed that costs within 1 year after an HF-related encounter were markedly higher for patients who died versus those who survived during that period. The cost difference occurred despite the truncated postindex period for those who died, and the largest cost driver was hospitalizations [33]. Given that mortality is associated with increased cost, the present study likely underestimates costs during the worsening assessment period by including only patients with 12 months continuous enrollment after HFrEF diagnosis.

Developing worsening HF has been associated with increased HCRU and associated costs [12]. A real-world study of in-hospital patients with worsening HF from the Acute Decompensated Heart Failure National Registry (ADHERE) reported an increased risk of all-cause and HFrelated readmissions at 30 days and 1 year, with associated increase in Medicare costs [12]. In that study, worsening HF was studied among already hospitalized patients and was defined as a need for therapy escalation at least $12 \mathrm{~h}$ after hospitalization, including initiation of inotropic medications or an intravenous dilator more than $12 \mathrm{~h}$ after hospitalization, transfer to intensive care, or treatment with advanced medical therapy after the first hospitalization day. The mean Medicare payments after discharge for patients with worsening HF were $\$ 6510$ at 30 days and $\$ 35,159$ at 1 year [12]. Even though patients with worsening HF were identified differently, the overall findings of higher costs and hospitalizations with an inhospital worsening event are consistent with the results of the present study.

Although existing cost studies have focused on the overall burden of HF, this study quantified the long-term clinical and economic burden of patients with chronic HFrEF following a WHFE versus patients with stable chronic HFrEF and highlighted the importance of optimizing the management of this population. Despite 
detailed clinical guidelines on treating patients with chronic HFrEF, along with newer therapies [21], there is still an unmet need for treatments or management strategies that can prevent or delay further HF events and the incremental costs associated with the events [34].

\section{Limitations}

The study was subject to limitations of the patient population studied and of the data acquired from the claims databases. The study included patients according to the presence of systolic HF ICD-10 codes, which could have included undiagnosed, underdiagnosed, or misdiagnosed patients with HFrEF [35]. Identification of eligible patients via ICD codes did not include a measurement of LVEF, so we do not know the actual EF values for the study population. The data analyzed were from claims used for billing purposes and may be subject to data errors, such as miscoding of medication; but errors of this type would apply to both cohorts assessed in this study. The study population was limited to commercially insured patients aged younger than 65 and focused only on patients who were fully covered by private insurance, which means further study is warranted to investigate whether similar trends exist among older patients and those with other types of coverage, including Medicare and Medicaid.

\section{CONCLUSIONS}

A retrospective claims-based study of commercially insured adult patients less than 65 years old showed that unadjusted and adjusted HCRU and costs were higher for patients with chronic HFrEF following a WHFE versus patients with stable chronic HFrEF during a 12-month worsening assessment period and continued to be higher for an additional 12 months after this period. The study shows that there is still a significant unmet need among patients with chronic HFrEF following a WHFE compared to patients with chronic HFrEF who remain stable. Therefore, prevention and/or treatment following a WHFE has the potential to reduce increased HCRU and costs, which themselves are associated with deleterious outcomes, including mortality.

\section{ACKNOWLEDGEMENTS}

Funding. The study, the journal's rapid service and open access fees were funded by Merck Sharp \& Dohme Corp., a subsidiary of Merck \& Co., Inc., Kenilworth, NJ, USA.

Authorship. All named authors meet the International Committee of Medical Journal Editors (ICMJE) criteria for authorship for this article, take responsibility for the integrity of the work as a whole, and have given their approval for this version to be published.

Medical Writing and Editorial Assistance. Medical writing was provided by Dr. Zachary Harrelson, PhD, of JK Associates, Inc., a member of Fishawack Health, Conshohocken, PA, USA. This assistance was funded by Merck Sharp \& Dohme Corp., a subsidiary of Merck \& Co., Inc., Kenilworth, NJ, USA.

Prior presentation. Data were presented at the ACC 2020 Annual Meeting (ACC 2020), Chicago, IL, March 28-30, 2020 and at the AMCP Managed Care \& Specialty Pharmacy 2020 Annual Meeting (AMCP 2020), Houston, TX, April 21-24, 2020.

Disclosures. Javed Butler has received research support from the NIH, Patient-Centered Outcomes Research Institute, and the EU; and serves as a consultant for Abbott, Adrenomed, Amgen, Array, AstraZeneca, Bayer, Boehringer Ingelheim, BMS, CVRx, Innolife, Janssen, LinaNova, Luitpold, Medtronic, Merck, Novartis, Novo Nordisk, Relypsa, Roche, Sanofi, V-Wave, and Vifor. Laurence M. Djatche, Lingfeng Yang, Joanne Brady, and Mei Yang are fulltime employees of Merck Sharp \& Dohme Corp., a subsidiary of Merck \& Co., Inc., Kenilworth, NJ, USA. Baanie Sawhney and Sreya Chakladar are full-time employees of Complete Health Economics and Outcomes Research 
Solutions and were contracted by Merck and Co. to conduct the study.

Compliance with Ethics Guidelines. This article reports on a retrospective, observational study using de-identified anonymized patientlevel data. Therefore, informed consent and ethics committee approval were not required. Merck accessed the IBM ${ }^{\circledR}$ MarketScan ${ }^{\circledR}$ Research Databases through a standard license agreement with IBM. Personnel from Complete Health Economics and Outcomes Research Solutions were granted access to the data via a third-party agreement in accordance with the terms and conditions of the agreement between Merck and IBM.

Data availability. The datasets analyzed during the current study are not publicly available because they were accessed under a standard license agreement with IBM.

Open Access. This article is licensed under a Creative Commons Attribution-NonCommercial 4.0 International License, which permits any non-commercial use, sharing, adaptation, distribution and reproduction in any medium or format, as long as you give appropriate credit to the original author(s) and the source, provide a link to the Creative Commons licence, and indicate if changes were made. The images or other third party material in this article are included in the article's Creative Commons licence, unless indicated otherwise in a credit line to the material. If material is not included in the article's Creative Commons licence and your intended use is not permitted by statutory regulation or exceeds the permitted use, you will need to obtain permission directly from the copyright holder. To view a copy of this licence, visit http://creativecommons.org/licenses/by$\mathrm{nc} / 4.0 /$.

\section{REFERENCES}

1. Benjamin EJ, Virani SS, Callaway CW, et al. Heart disease and stroke statistics-2018 update: a report from the American Heart Association. Circulation. 2018;137(12):e67-492.
2. Desai AS, Stevenson LW. Rehospitalization for heart failure: predict or prevent? Circulation. 2012;126(4):501-6.

3. Jackson SL, Tong X, King RJ, et al. National burden of heart failure events in the United States, 2006 to 2014. Circ Heart Fail. 2018;11(12):e004873.

4. Lin AH, Chin JC, Sicignano NM, Evans AM. Repeat hospitalizations predict mortality in patients with heart failure. Mil Med. 2017;182(9):e1932-7.

5. Gerber Y, Weston SA, Redfield MM, et al. A contemporary appraisal of the heart failure epidemic in Olmsted County, Minnesota, 2000 to 2010. JAMA Intern Med. 2015;175(6):996-1004.

6. Shah KS, Xu H, Matsouaka RA, et al. Heart failure with preserved, borderline, and reduced ejection fraction: 5-year outcomes. J Am Coll Cardiol. 2017;70(20):2476-86.

7. Gurwitz JH, Magid DJ, Smith DH, et al. Contemporary prevalence and correlates of incident heart failure with preserved ejection fraction. Am J Med. 2013;126(5):393-400.

8. Tsao CW, Lyass A, Enserro D, et al. Temporal trends in the incidence of and mortality associated with heart failure with preserved and reduced ejection fraction. JACC Heart Fail. 2018;6(8):678-85.

9. Ho JE, Lyass A, Lee DS, et al. Predictors of new-onset heart failure: differences in preserved versus reduced ejection fraction. Circ Heart Fail. 2013;6(2): 279-86.

10. Vasan RS, Xanthakis V, Lyass A, et al. Epidemiology of Left Ventricular Systolic Dysfunction and Heart Failure in the Framingham Study: an echocardiographic study over 3 decades. JACC Cardiovasc Imaging. 2018;11(1):1-11.

11. Ibrahim NE, Song Y, Cannon CP, et al. Heart failure with mid-range ejection fraction: characterization of patients from the PINNACLE Registry ${ }^{\circledR}$. ESC Heart Fail. 2019;6(4):784-92.

12. DeVore AD, Hammill BG, Sharma PP, et al. Inhospital worsening heart failure and associations with mortality, readmission, and healthcare utilization. J Am Heart Assoc. 2014;3(4):e001088.

13. Ambrosy AP, Fonarow GC, Butler J, et al. The global health and economic burden of hospitalizations for heart failure: lessons learned from hospitalized heart failure registries. J Am Coll Cardiol. 2014;63(12):1123-33.

14. Butler J, Yang M, Manzi MA, et al. Clinical Course of patients with worsening heart failure with 
reduced ejection fraction. J Am Coll Cardiol. 2019;73(8):935-44.

15. Okumura N, Jhund PS, Gong J, et al. Importance of clinical worsening of heart failure treated in the outpatient setting: evidence from the Prospective Comparison of ARNI with ACEI to Determine Impact on Global Mortality and Morbidity in Heart Failure Trial (PARADIGM-HF). Circulation. 2016;133(23):2254-62.

16. Skali H, Dwyer EM, Goldstein R, et al. Prognosis and response to therapy of first inpatient and outpatient heart failure event in a heart failure clinical trial: MADIT-CRT. Eur J Heart Fail. 2014;16(5):560-5.

17. Setoguchi S, Stevenson LW, Schneeweiss S. Repeated hospitalizations predict mortality in the community population with heart failure. Am Heart J. 2007;154(2):260-6.

18. Solomon SD, Dobson J, Pocock S, et al. Influence of nonfatal hospitalization for heart failure on subsequent mortality in patients with chronic heart failure. Circulation. 2007;116(13):1482-7.

19. ENTRESTO $^{\circledR}$ (sacubitril and valsartan) [prescribing information]. Novartis Pharmaceuticals Corporation, October 2019. https://www.pharma.us. novartis.com/sites/www.pharma.us.novartis.com/ filesentresto.pdf. Accessed 15 Apr 2020.

20. CORLANOR ${ }^{\circledR}$ (ivabradine) [prescribing information]. Amgen Inc., April 2019. https://www.pi. amgen.com/ /media/amgen/repositorysites/piamgen-com/corlanor/corlanor_pi_hcp.pdf. Accessed 15 Apr 2020.

21. Yancy CW, Jessup M, Bozkurt B, et al. 2017 ACC/ AHA/HFSA focused update of the 2013 ACCF/AHA guideline for the management of heart failure: a report of the American College of Cardiology/ American Heart Association Task Force on Clinical Practice Guidelines and the Heart Failure Society of America. J Card Fail. 2017;23(8):628-51.

22. Hidalgo FJ, Anguita M, Castillo JC, et al. Effect of early treatment with ivabradine combined with beta-blockers versus beta-blockers alone in patients hospitalised with heart failure and reduced left ventricular ejection fraction (ETHIC-AHF): a randomised study. Int J Cardiol. 2016;217:7-11.

23. Solomon SD, Claggett B, Desai AS, et al. Influence of ejection fraction on outcomes and efficacy of sacubitril/valsartan (LCZ696) in heart failure with reduced ejection fraction: the Prospective comparison of ARNI with ACEI to Determine Impact on Global Mortality and Morbidity in Heart Failure (PARADIGM-HF) trial. Circ Heart Fail. 2016;9(3): e002744.
24. Nichols GA, Reynolds K, Kimes TM, Rosales AG, Chan WW. Comparison of risk of re-hospitalization, all-cause mortality, and medical care resource utilization in patients with heart failure and preserved versus reduced ejection fraction. Am J Cardiol. 2015;116(7):1088-92.

25. Olchanski N, Vest AR, Cohen JT, Neumann PJ, DeNofrio D. Cost comparison across heart failure patients with reduced and preserved ejection fractions: analyses of inpatient decompensated heart failure admissions. Int J Cardiol. 2018;261:103-8.

26. Stålhammar J, Stern L, Linder R, et al. Resource utilization and cost of heart failure associated with reduced ejection fraction in Swedish patients. J Med Econ. 2012;15(5):938-46.

27. FRED Federal Reserve Bank of St. Louis. U.S. Bureau of Labor Statistics, consumer price index for all urban consumers: medical care in U.S. city average [CUUSO000SAM]. https://fred.stlouisfed.org/series/ CUUSO000SAM. Accessed 15 Apr 2020.

28. Brusselaers N, Lagergren J. The Charlson Comorbidity Index in registry-based research. Methods Inf Med. 2017;56(5):401-6.

29. Quan H, Sundararajan V, Halfon P, et al. Coding algorithms for defining comorbidities in ICD-9-CM and ICD-10 administrative data. Med Care. 2005;43(11):1130-9.

30. Chipeta MG, Ngwira BM, Simoonga C, Kazembe LN. Zero adjusted models with applications to analysing helminths count data. BMC Res Notes. 2014;7:856.

31. Gilligan AM, Franchino-Elder J, Song $\mathrm{X}$, et al. Comparison of all-cause costs and healthcare resource use among patients with newly-diagnosed non-valvular atrial fibrillation newly treated with oral anticoagulants. Curr Med Res Opin. 2018;34(2):285-95.

32. Obi EN, Swindle JP, Turner SJ, Russo PA, Altan A. Health care costs for patients with heart failure escalate nearly 3-fold in final months of life. J Manag Care Spec Pharm. 2016;22(12):1446-56.

33. Obi EN, Swindle JP, Turner SJ, Russo PA, Altan A. Healthcare costs among patients with heart failure: a comparison of costs between matched decedent and survivor cohorts. Adv Ther. 2017;34(1):261-76.

34. Bress AP, King JB, Brixner D, et al. Pharmacotherapy treatment patterns, outcomes, and health resource utilization among patients with heart failure with reduced ejection fraction at a U.S. academic medical center. Pharmacotherapy. 2016;36(2):174-86. 
35. McCormick N, Lacaille D, Bhole V, Avina-Zubieta JA. Validity of heart failure diagnoses in administrative databases: a systematic review and meta-analysis. PLoS One. 2014;9(8):e104519. 